\title{
Protective effects of icariin-mediated SIRT1/FOXO3 signaling pathway on intestinal ischemia/reperfusion-induced acute lung injury
}

\author{
FENG ZHANG $^{1 *}$, ZHEN-LU LI ${ }^{1 *}$, XIAO-MEI XU ${ }^{2}$, YAN HU $^{2}$, JI-HONG YAO ${ }^{2}$, WEI XU ${ }^{1}$, \\ HUI-RONG JING ${ }^{1}$, SHU WANG ${ }^{1}$, SHI-LI NING ${ }^{1}$ and XIAO-FENG TIAN ${ }^{1}$
}

${ }^{1}$ Department of General Surgery, The Second Affiliated Hospital of Dalian Medical University, Dalian, Liaoning 116023;

${ }^{2}$ Department of Pharmacology, Dalian Medical University, Dalian, Liaoning 116044, P.R. China

Received September 22, 2013; Accepted May 15, 2014

DOI: $10.3892 / \mathrm{mmr} .2014 .2679$

\begin{abstract}
Acute lung injury (ALI) is a common complication following intestinal ischemia/reperfusion $(\mathrm{I} / \mathrm{R})$ and is a major contributing factor to its high mortality rate. Sirtuin 1 (SIRT1), a NAD ${ }^{+}$-dependent deacetylase, has been reported to have an important role in apoptosis inhibition, oxidative stress resistance and cell lifespan extension through its deacetylation of forkhead box protein $\mathrm{O} 3$ (FOXO3). It has been demonstrated that icariin (ICA), a flavonoid extracted from Epimedium, upregulates SIRT1 expression. The aim of the present study was to examine whether ICA-mediated SIRT1/FOXO3 signaling pathway activation had a protective effect on intestinal I/R-induced ALI. The effects of ICA on intestinal I/R-induced ALI and its regulation of the SIRT1/FOXO3 signaling pathway on intestinal I/R-induced ALI were investigated in rats. The results demonstrated that ICA pretreatment markedly reduced intestinal I/R-induced ALI as indicated by histological alterations, including decreased tumor necrosis factor- $\alpha$ (TNF- $\alpha$ ), interleukin 6 (IL-6), reduced oxidative stress, acetylated FOXO3 and B-cell lymphoma 2 (Bcl-2)-interacting mediator of cell death levels, and increased glutathione (GSH), GSH peroxidase, SIRT1, manganese superoxide dismutase and Bcl-2 levels in rat lung tissues. Furthermore, ICA pretreatment upregulated SIRT1 expression, which then downregulated FOXO3 acetylation. In conclusion, ICA exhibited significant protective effects in intestinal I/R-induced ALI. The protective effect of ICA may
\end{abstract}

Correspondence to: Professor Xiao-Feng Tian, Department of General Surgery, The Second Affiliated Hospital of Dalian Medical University, 467 Zhongshan Road, Dalian, Liaoning 116023, P.R. China

E-mail:txfdl@hotmail.com

*Contributed equally

Key words: icariin, intestinal ischemia/reperfusion, acute lung injury, sirtuin 1, forkhead box protein $\mathrm{O} 3$ be attributed to the upregulation of SIRT1, which contributed to FOXO3 deacetylation and the modulation of downstream antioxidative and anti-apoptotic factors.

\section{Introduction}

Ischemia/reperfusion ( $\mathrm{I} / \mathrm{R})$ injury of the intestine is a common type of intraoperative tissue and organ damage that is secondary to vascular disease of the gut and its mesentery, as well as to a variety of clinical pathophysiological processes of the disease, including acute blood loss, shock, severe trauma, small bowel transplantation and sepsis, among others $(1,2)$. I/R injury is characterized by a high mortality rate of up to $60-80 \%$ (3) and causes gut barrier disruption, oxygen free radical generation and endotoxin entry into the circulation, thereby leading to severe systemic inflammation and eventual multiple organ failure of the lungs, liver and kidneys (4-7). In fact, acute lung injury (ALI) is a common complication following intestinal I/R and tends to develop as acute respiratory distress syndrome, which is a major contributor to its high mortality rate (8-11).

Specific mechanisms underlying ALI, however, are highly complex and involve the production of reactive oxygen species, the activation of cytokines and inflammatory mediators, and the initiation of cell apoptosis $(12,13)$. A previous study reported that the antioxidant manganese superoxide dismutase (MnSOD) and the apoptosis-associated molecule B-cell lymphoma 2 (Bcl-2) have important roles in the pathogenesis of I/R-induced ALI in the small intestine, in which the above molecules are mediated by the regulatory factor forkhead box protein $\mathrm{O} 3$ (FOXO3) (14). Furthermore, the sirtuin 1 (SIRT1)/FOXO3 signaling pathway is indispensable to the antioxidative and anti-apoptotic properties of I/R disease (15).

SIRT1, a nicotinamide adenine dinucleotide (NAD)-dependent class III histone deacetylase (HDAC), is a mammalian SIR2 homologue in the yeast Saccharomyces cerevisiae. Among the seven sirtuin genes (SIRT1-7) in mammals, SIRT1 is the most widely studied and was found to be mostly localized to the nucleus, where it has a vital role in transcriptional repression via histone deacetylation (16-18). Recent studies have identified that SIRT1 is important in DNA damage repair, apoptosis inhibition, oxidative stress 
resistance and cell lifespan extension (19). According to a study by Hsu et al (20), SIRT1 expression in myocardial I/R injury may confer a protective effect. For example, SIRT1 regulates a diverse set of proteins, including p53 and the transcription factors nuclear factor-kappa $\mathrm{B}(\mathrm{NF}-\mathrm{\kappa} \mathrm{B})$ and $\mathrm{FOXO}$, and promotes cell survival in response to severe stress (21-24). Under ischemic or hypoxic conditions, FOXO3 may enhance cellular resistance to oxidative stress through SIRT1 deacetylation in a process that is mainly completed by MnSOD, which is able to scavenge reactive oxygen species (ROS) $(21,25)$. In addition, a study by Motta et al (24) demonstrated that mammalian SIRT1 deacetylated FOXO3 and attenuated its pro-apoptotic abilities. Furthermore, Brunet et al (21) revealed that SIRT1 deacetylation increased the ability of FOXO3 to induce cell cycle arrest and oxidative stress resistance; however, this inhibited the ability of FOXO3 to induce cell death. Therefore, to the best of our knowledge, the present study assessed, for the first time, whether SIRT1 may possess protective effects on intestinal I/R-induced ALI by regulating the FOXO3 signaling pathway.

A variety of natural products are involved in SIRT1 regulation, including polyphenols and flavonoids, among others. Resveratrol is a representative polyphenolic compound that is known to have a role in SIRT1 regulation. Icariin (ICA) is a flavonoid extracted from Epimedium, a genus of flowering plants in the family Berberidaceae, which is used in Traditional Chinese Herbal Medicine as an aphrodisiac. A recent study by Wang et al (26) found that ICA upregulated SIRT1 expression, which resulted in the enhancement of neuronal viability and the suppression of neuronal death in response to oxygen and glucose deprivation. Furthermore, regulation of the SIRT1 signaling pathway by ICA has been demonstrated to ameliorate oxidative stress in the treatment of central nervous system disorders $(27,28)$. However, to the best of our knowledge, no studies have reported ICA regulation of the SIRT1/FOXO3 signaling pathway in intestinal I/R injury.

Therefore, the aims of the present study were i) to determine whether ICA-mediated SIRT1/FOXO3 activation had a protective effect on intestinal I/R-induced ALI by inhibition of alveolar cell apoptosis and increased antioxidant enzyme activity and ii) to determine whether ICA was able to increase SIRT1 levels to reduce the content of acetylated FOXO3 and regulate the antioxidant $\mathrm{MnSOD}$ and apoptosis-associated factors, Bcl-2 and Bcl-2 interacting mediator of cell death (Bim).

\section{Materials and methods}

Animals and reagents. Male Sprague-Dawley rats (aged 5 weeks) weighing 160-180 g were obtained from the Animal Center of Dalian Medical University (Dalian, China) and maintained in a temperature-controlled $\left(25^{\circ} \mathrm{C}\right)$ facility with a $12-\mathrm{h}$ light/12-h dark cycle with free access to a standard laboratory diet and water. The rats were acclimatized for one week prior to the experiments. All procedures were conducted according to the Institutional Animal Care Guidelines and were approved by the Institutional Ethics Committee of Dalian Medical College (Dalian, China). ICA ( $>98 \%$ of purity) was purchased from Shanghai Winherb Medical S\&T Development Co., Ltd. (Shanghai, China).
Intestinal I/R model and experimental design. The rat intestinal I/R model was established as previously described with several small modifications (29). Briefly, the superior mesenteric artery (SMA) was identified through a midline laparotomy and occluded gently by an atraumatic microvascular clamp for $60 \mathrm{~min}$. The occlusion was confirmed when the mesenteric pulsations ceased and the intestines became pale. A 120-min reperfusion was then performed. In the control groups, the rats underwent surgical preparation, including isolation of the SMA but without occlusion. All of the animals were randomly divided into five groups, with eight rats in each of the following: i) Control group; ii) intestinal I/R group; iii) control+ICA group, where the mice were pretreated with ICA $(60 \mathrm{mg} / \mathrm{kg}$ by intragastric administration for three consecutive days); iv) I/R+ICA group, where the mice were pretreated with ICA as above and then surgery was performed in analogy to that in the I/R group and v) $\mathrm{I} / \mathrm{R}+\mathrm{ischemia}$ preconditioning (IPC) group, where the rats were pretreated with reperfusion for 5 min following occlusion of the SMA for $5 \mathrm{~min}$, which was repeated three times and then surgery was performed in analogy to that in the I/R group. All of the animals were sacrificed at the end of the reperfusion, and the tissues and blood samples were obtained for analysis.

Assessment of lung histopathology. The isolated right middle lobe of the lung was fixed in $4 \%$ formaldehyde. After being embedded in paraffin, the tissues were sectioned and stained with hematoxylin and eosin (H\&E). The specimens were examined under light microscopy (Leica DM4000, Wetzlar, Germany). The pathological scores of lung damage were examined according to the previously described methods (30).

Measurement of serum cytokine levels. Blood samples were collected from the abdominal aorta and allowed to coagulate for $2 \mathrm{~h}$ on ice. The serum was then isolated as the supernatant fraction following centrifugation at 3,000 $\mathrm{x} \mathrm{g}$ for $20 \mathrm{~min}$. Tumor necrosis factor- $\alpha$ (TNF- $\alpha$ ) and interleukin 6 (IL-6) levels were measured with ELISA kits (Wuhan Boster Biological Technology, Ltd., Wuhan, China), according to the manufacturer's instructions.

Lung malondialdehyde assay (MDA), glutathione (GSH) and $G S H$ peroxidase $(G S H-P X)$ activity assay. The lung tissues were weighed, minced and transferred into a centrifuge tube. The 1:9 (w/v) volume of cold isotonic sodium chloride solution was added and the mixtures were homogenized by a tissue disperser (IKA, Staufen, Germany) four times at $4^{\circ} \mathrm{C}$, each for $10 \mathrm{sec}$. The homogenates were centrifuged at 3,000 x $\mathrm{g}$ for $15 \mathrm{~min}$ to remove the debris. The MDA, GSH and GSH-PX activity in the supernatants were determined using an assay kit (Nanjing Jiancheng Bioengineering Institute, Nanjing, China), according to the manufacturer's instructions.

Terminal deoxynucleotidyl transferase-mediated dUTP nick end labeling (TUNEL) assay. The TUNEL assays were performed with the TMR red In Situ Cell Death Detection kit (supplemented with $0.1 \%$ sodium citrate; Hoffmann-La Roche Inc., Nutley, NJ, USA), according to the manufacturer's instructions. The lung samples were treated as mentioned in the histopathological analysis. Briefly, the lung tissue 
sections were pretreated with $0.1 \%$ Triton $\mathrm{X}-100$ for $8 \mathrm{~min}$, washed with PBS, then stained by TUNEL reaction mixture (label and enzyme solutions) for $1 \mathrm{~h}$ at $37^{\circ} \mathrm{C}$. The fluorescein isothiocyanate-labeled TUNEL-positive cells were imaged under a fluorescent microscope (DMI 4000 B; Leica, Wetzlar, Germany) using $488 \mathrm{~nm}$ excitation and $530 \mathrm{~nm}$ emission. The cells exhibiting green fluorescence were defined as apoptotic cells.

Western blot analysis of SIRT1, FOXO3, MnSOD, Bim and Bcl-2 in lung tissue. Nuclear and cytosolic proteins were extracted from the lung tissues with a protein extraction kit (Nanjing KeyGen Biotech. Co. Ltd., Nanjing, China) according to the manufacturer's instructions. The protein concentrations were determined using an bicinchoninic acid protein assay kit (Beyotime Institute of Biotechnology, Jiangsu, China). Equal amounts of nuclear and cytosolic protein were used to determine SIRT1, FOXO3, MnSOD, Bim and Bcl-2 protein concentrations. The sample proteins $(50 \mu \mathrm{g} /$ lane $)$ were separated by $12 \%$ SDS-PAGE (Bio-Rad, Hercules, CA, USA) and then transferred to a polyvinylidene difluoride membrane (Millipore, Bedford, MA, USA). Following blocking, the membranes were probed with the following primary antibodies: Anti-SIRT1 (mouse, mAb, 1:4000, Abcam Ltd., Cambridge, UK), anti-FOXO3 (rabbit, pAb, 1:2000, Abcam Ltd.), anti-MnSOD (mouse, mAb, 1:1000, Abcam Ltd.), anti-Bcl-2 (rabbit, pAb, 1:1000, Beyotime Institute of Biotechnology) and anti- $\beta$-actin (mouse, mAb, 1:1000, Santa Cruz Biotechnology, Inc., Santa Cruz, CA, USA) overnight at $4^{\circ} \mathrm{C}$. Following washing in tris-buffered saline containing Tween 20 (TTBS) three times, the membranes were incubated with the relevant horseradish peroxidase-conjugated secondary antibodies for $2 \mathrm{~h}$ at $37^{\circ} \mathrm{C}$. Following adequate washing with TTBS, the membranes were exposed to chemiluminescence-plus reagents. The blots were then developed with a BioSpectrum-410 multispectral imaging system with a Chemi HR camera 410 and the density of the bands was determined using Gel-Pro Analyzer Version 4.0 (Media Cybernetics, Bethesda, MD, USA).

Immunoprecipitation assay. The protein samples were extracted from the lung tissues, mixed with non-specific rabbit immunoglobulin $G$ and the fully resuspended Protein $A+G$ Agarose (Beyotime Institute of Biotechnology) and slowly shaken at $4^{\circ} \mathrm{C}$ for $2 \mathrm{~h}, 1,000 \mathrm{x}$ g for $5 \mathrm{~min}$ and the supernatant was used for subsequent immunoprecipitation. Rabbit anti-human polyclonal acetylated lysine antibody (Abcam Ltd.) was added at $4{ }^{\circ} \mathrm{C}$, the mixture was slowly agitated for $18 \mathrm{~h}$ and then fully resuspended in Protein A+G Agarose with $4^{\circ} \mathrm{C}$ with gentle agitation for $2 \mathrm{~h}, 1,000 \mathrm{x}$ g for $5 \mathrm{~min}$, and then the precipitation of all of the acetylated protein was performed. Finally, acetylated FOXO3 proteins were detected using western blot analysis with the previously mentioned antibodies against FOXO3 (Abcam Ltd.).

RNA isolation and polymerase chain reaction (PCR) analysis. Total RNA was extracted from the frozen lung using RNA iso Plus (Takara Biotechnology (Dalian) Co., Ltd., Dalian, China) following the manufacturer's instructions. RNA was dissolved in diethylpyrocarbonate-treated deionized water, and the RNA concentrations were analyzed using a nanodrop spectrophotometer (UV1102II, Shanghai, China) to determine quality and quantity. Reverse transcription was performed using a Takara RNA PCR kit (AMV) version 3.0 (Takara Bio, Inc., Shiga, Japan). For PCR analysis, the primers and their sequences were as follows: SIRT1 (forward, F) 5'-GCAACAGCATCTTGCCTGAT-3' and (reverse, R) 5'-GTGCTACTGGTCTCACTT-3'; $\beta$-actin, F 5'-AGAGGGAAATCGTGCGTGAC-3' and R 5'-CAATAGTGATGACCTGGCCGT-3'. Two-step PCR was conducted according to the instructions of the Takara RNA PCR Kit (AMV) version 3.0. The conditions for reverse transcription were $42^{\circ} \mathrm{C}$ for $30 \mathrm{~min}, 99^{\circ} \mathrm{C}$ for $5 \mathrm{~min}$ and then $5^{\circ} \mathrm{C}$ for $5 \mathrm{~min}$ to terminate the reverse transcription. The PCR was performed as follows: $94^{\circ} \mathrm{C}$ for $30 \mathrm{sec}$ for denaturation; annealing, $55^{\circ} \mathrm{C}$ for $30 \mathrm{sec}$ and $72^{\circ} \mathrm{C}$ for $90 \mathrm{sec}$ for extension. The products were then separated by electrophoresis using $1.5 \%$ agarose gels. The bands were visualized using the BioSpectrum-410 multispectral imaging system with a Chemi HR camera 410 (UVP, Upland, CA, USA).

Statistical analysis. The mean \pm standard deviation values were calculated to summarize all of the measurements. A one-way analysis of variance was used to compare the means of the five groups. The Student-Newman-Keuls'/Least Significant Difference test was performed to compare all pair means. A value of $\mathrm{P}<0.05$ was considered to indicate a statistically significant difference. All statistical analyses were run with the SPSS version 17 Statistical Software Package (SPSS, Inc., Chicago, IL, USA).

\section{Results}

Effects of ICA on intestinal I/R-induced ALI and systemic inflammation. To determine whether ICA protects against intestinal I/R-induced ALI, the histopathological properties of lung tissue, serum TNF- $\alpha$ and IL-6 levels, as well as GSH and GSH-PX activity in the lung were examined. As demonstrated in Fig. 1A, in the I/R group, the lung tissue was markedly damaged, as characterized by severe congestive alveoli, alveolar wall telangiectasia, increased inflammatory cell infiltration, thickened alveolar walls and hyaline membrane formation. In conjunction with this damage, serum TNF- $\alpha$ and IL-6 levels were significantly increased, while lung GSH and GSH-PX activities were decreased (Table I). By contrast, in the I/R+ICA and IPC groups, the results demonstrated that ICA and IPC attenuated inflammatory infiltration, improved lung histopathology scores (Fig. 1B), reduced serum TNF- $\alpha$ and IL-6 levels, and increased lung GSH and GSH-PX activities (Table I). These results indicated that ICA offered effective protection against intestinal I/R-induced ALI.

Effects of ICA on the SIRT1-FOXO3 pathway activation in rat lung tissues. To determine whether intestinal I/R, ICA administration and IPC affected SIRT1 mRNA expression in the lung, PCR analysis of SIRT1 was performed. As demonstrated in Fig. 2A, following intestinal ischemia for $60 \mathrm{~min}$ followed by reperfusion for $120 \mathrm{~min}$, SIRT1 mRNA expression in the injured lung tissue was significantly decreased compared with that in the control tissues. Following ICA administration or IPC, SIRT1 mRNA expression was significantly increased 
Table I. GSH, GSH-PX, TNF- $\alpha$ and IL-6 expression levels in intestinal tissues from the different groups (mean \pm standard deviation, $n=8$ ).

\begin{tabular}{lcccc}
\hline Group & GSH $(\mathrm{mg} / \mathrm{g}$ protein) & GSH-PX $(\mathrm{U} / \mathrm{g}$ protein) & TNF- $\alpha(\mathrm{pg} / \mathrm{ml})$ & $\mathrm{IL}-6(\mathrm{pg} / \mathrm{ml})$ \\
\hline Sham & $6.32 \pm 0.51$ & $87.51 \pm 6.54$ & $63.96 \pm 10.12$ & $165.72 \pm 21.47$ \\
I/R & $2.54 \pm 0.31^{\mathrm{a}}$ & $36.27 \pm 3.78^{\mathrm{a}}$ & $117.05 \pm 15.31^{\mathrm{a}}$ & $257.88 \pm 34.97^{\mathrm{a}}$ \\
Sham+ICA & $6.48 \pm 0.42$ & $95.74 \pm 7.78$ & $62.31 \pm 10.77$ & $151.89 \pm 18.25$ \\
I/R+ICA & $4.53 \pm 0.57^{\mathrm{b}}$ & $65.27 \pm 10.53^{\mathrm{b}}$ & $83.81 \pm 9.69^{\mathrm{b}}$ & $205.96 \pm 21.87^{\mathrm{b}}$ \\
I/R+IPC & $3.70 \pm 0.46^{\mathrm{b}}$ & $55.53 \pm 8.30^{\mathrm{b}}$ & $99.70 \pm 12.26^{\mathrm{b}}$ & $200.73 \pm 20.91^{\mathrm{b}}$ \\
\hline
\end{tabular}

${ }^{\mathrm{a}} \mathrm{P}<0.01$ vs. the sham group; ${ }^{\mathrm{b}} \mathrm{P}<0.01$ vs. the I/R group. GSH, glutathione; GSH-PX, GSH peroxidase; TNF- $\alpha$, tumor necrosis factor- $\alpha$; IL-6, interleukin 6; I/R, ischemia/reperfusion; ICA, icariin.
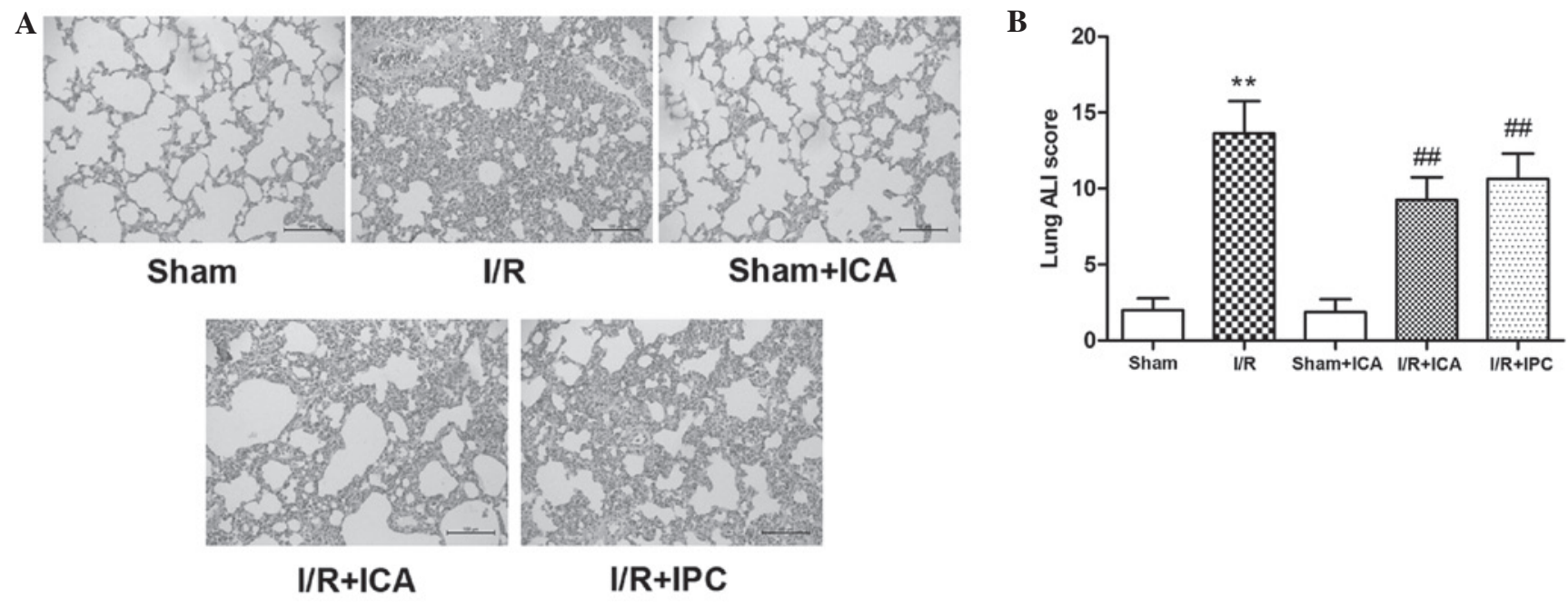

Figure 1. ICA improved intestinal I/R-induced lung histopathological properties. (A) Pathological changes in the lung tissue from each group (magnification, $\mathrm{x} 400)$; (B) normalized histopathological properties of the lung tissues. Values are presented as the mean \pm standard deviation ( $=8$ ). ${ }^{* *} \mathrm{P}<0.01$ vs. the control group; ${ }^{\#} \mathrm{P}<0.01$ vs. the I/R group. I/R, ischemia/reperfusion; ALI, acute lung injury; ICA, icariin; IPC, ischemia preconditioning.

compared with that in the I/R group. These results suggested that ICA administration or IPC increased SIRT1 mRNA expression in the I/R group.

To determine whether intestinal I/R, ICA administration or IPC affected the expression of proteins of the SIRT1-FOXO3 pathway in lung tissues, including SIRT1 and acetylated FOXO3, western blot analysis for SIRT1 and immunoprecipitation of acetylated FOXO3 were performed. Brunet et al (21) reported that in response to oxidative stress, the deacetylase SIRT1 forms a protein complex with the forkhead transcription factor FOXO3 that contributes to FOXO3 deacetylation and then steers FOXO3-dependent responses away from cell death and towards stress resistance. As demonstrated in Fig. 2B, following intestinal ischemia for $60 \mathrm{~min}$ and subsequent reperfusion for $120 \mathrm{~min}$, SIRT1 protein expression in the lung tissue was significantly decreased compared with that in the controls, while acetylated FOXO3 was significantly increased. Following ICA administration or IPC, SIRT1 expression was increased, whereas acetylated FOXO3 expression was significantly decreased compared with the I/R group. Collectively, these results suggested that both ICA administration and IPC suppressed intestinal I/R-induced ALI via upregulation of SIRT1 expression, thereby contributing to FOXO3 deacetylation.
In conclusion, ICA administration and IPC promoted SIRT1 mRNA and protein expression, which contributed to FOXO3 deacetylation in intestinal I/R-induced ALI in rats.

Effects of ICA-mediated SIRT1-FOXO3 pathway activation on the expression of apoptosis-associated factors in rat lung tissue. Since it is well-established that intestinal I/R-induced ALI causes alveolar cell apoptosis, the TUNEL assay was performed to assess the extent of apoptosis. As revealed in Fig. 3A, marked alveolar cell apoptosis was observed in the lungs of rats in the intestinal I/R group, whereas in the ICA and IPC groups, alveolar cell apoptosis was markedly attenuated.

The pro-apoptotic factor Bim and anti-apoptotic factor Bcl-2 have crucial roles in the regulation of cellular responses to apoptosis. According to a study by Brunet et al (21), SIRT1 inhibited the ability of FOXO3 to induce cell death, whereas Motta et al (24) and Dijkers et al (31) demonstrated that SIRT1 deacetylated and repressed the activity of the forkhead transcription factor FOXO3a, which, in turn, reduced the expression of the forkhead-dependent apoptotic factor Bim. To determine whether intestinal I/R, ICA administration and IPC affected Bim and Bcl-2 expression in ALI, western blot analysis 
A
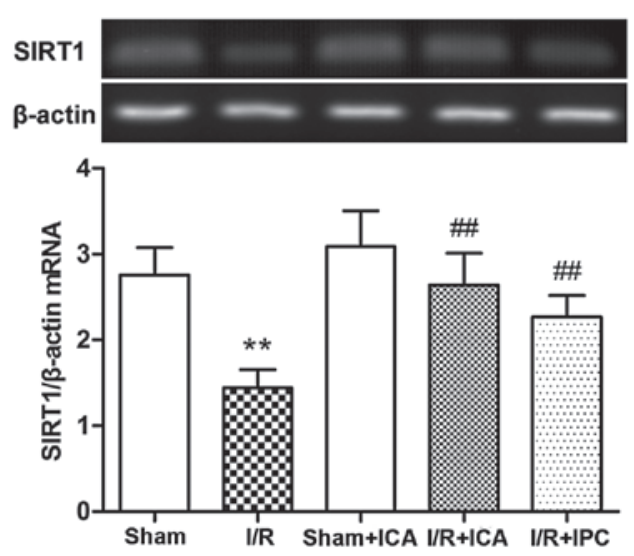

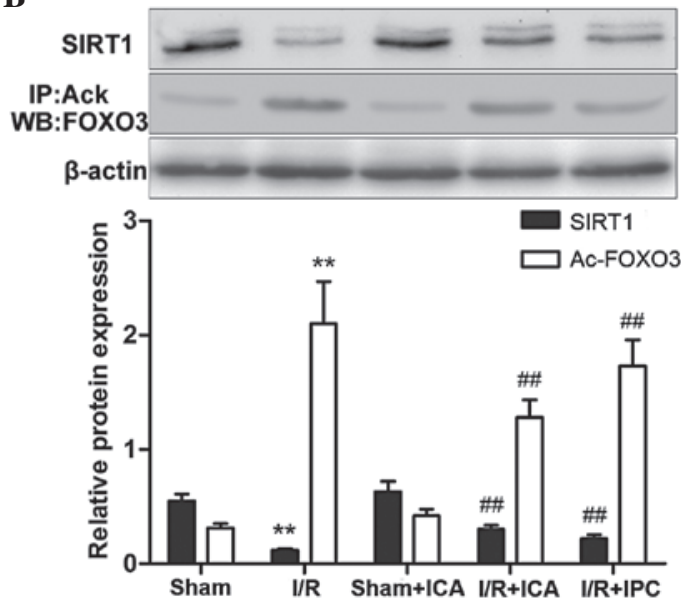

Figure 2. Effects of ICA on SIRT1-FOXO3 pathway activation in rat lung tissues. (A) Effects of ICA on SIRT1 mRNA expression in the rat lung tissues. ICA pretreatment or IPC increased SIRT1 mRNA expression in the lung following intestinal I/R. (B) Effects of ICA on SIRT1-FOXO3 pathway activation in the rat lung tissues. ICA pretreatment or IPC increased SIRT1 protein expression in the lung tissues following intestinal I/R, while it suppressed acetylated FOXO3 protein expression. Values are presented as the mean \pm standard deviation $(n=3){ }^{* *} \mathrm{P}<0.01$ vs. the control group; ${ }^{\# \#} \mathrm{P}<0.01$ vs. the $\mathrm{I} / \mathrm{R}$ group. $\mathrm{I} / \mathrm{R}$, ischemia/reperfusion; ICA, icariin; SIRT1, sirtuin 1; IP, immunoprecipitation assay; WB, western blot analysis; FOXO3, forkhead box protein O3; IPC, ischemia preconditioning.

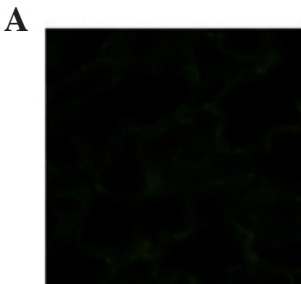

Sham

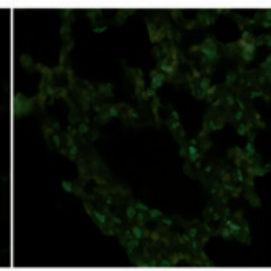

I/R

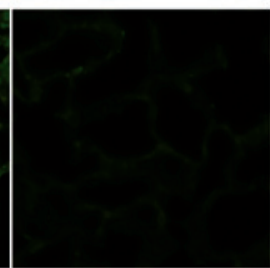

Sham+ICA

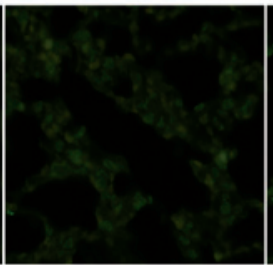

I/R+ICA

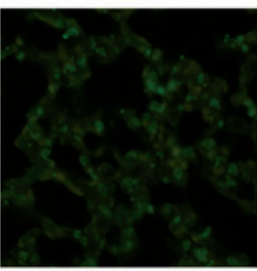

I/R+IPC

B
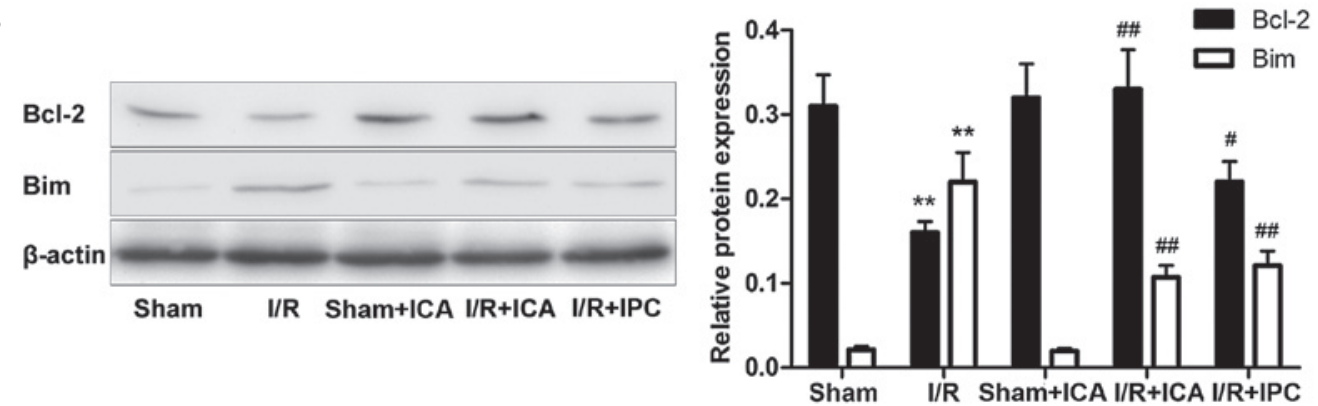

Figure 3. Effects of ICA-mediated SIRT1-FOXO3 pathway activation on the expression of apoptosis-associated factors in rat lung tissues. (A) ICA attenuated I/R-induced alveolar cell apoptosis in vivo, as confirmed using the TUNEL assay. The content of TUNEL-positive cells was equal to the amount of green fluorescenct in the image (magnification, x400). (B) Western blot analysis of Bim and Bcl-2 protein in lung tissue samples from each group. ICA pretreatment or IPC reduced Bim expression and enhanced Bcl-2 expression in the lung tissue samples following intestinal I/R. Values are presented as the mean \pm standard deviation ( $\mathrm{n}=3$ ). ${ }^{* *} \mathrm{P}<0.01$ vs. the control group; ${ }^{\#} \mathrm{P}<0.05,{ }^{\# \#} \mathrm{P}<0.01$ vs. the I/R group. I/R, ischemia/reperfusion; ICA, icariin; IPC, ischemia preconditioning; SIRT1, sirtuin 1; FOXO3, forkhead box protein O3; TUNEL, terminal deoxynucleotidyl transferase-mediated dUTP nick end labeling; Bcl-2, B-cell lymphoma 2; Bim, Bcl-2-interacting mediator of cell death.

was performed. As revealed in Fig. 3B, following intestinal ischemia for $60 \mathrm{~min}$ and subsequent reperfusion for $120 \mathrm{~min}$, the expression of Bim was elevated and Bcl-2 was significantly decreased in the injured lung tissues compared with those in the controls. Following ICA administration or IPC, Bim levels were decreased and Bcl-2 levels were increased compared with those in the I/R group. Along with the conclusions mentioned above, these phenomena indicated that ICA administration or IPC upregulated SIRT1 expression and the latter deacetylated FOXO3, thereby lowering the pro-apoptotic factor Bim, while enhancing the anti-apoptotic factor Bcl-2, which suggested that the ICA-mediated SIRT1-FOXO3 activation produced an anti-apoptotic effect in intestinal I/R-induced ALI.

Effects of ICA-mediated SIRT1-FOXO3 pathway activation on the expression of antioxidative factors in rat lung tissues. MDA is a degradation product of polyunsaturated fatty acid peroxidation and arachidonic acid metabolism, and is used as an indicator of oxidative stress in cells and tissues. Therefore, serum MDA levels were analyzed to quantify oxidative 
A

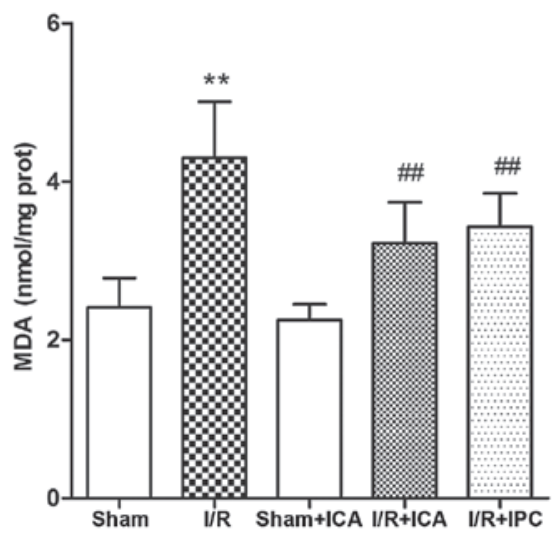

B

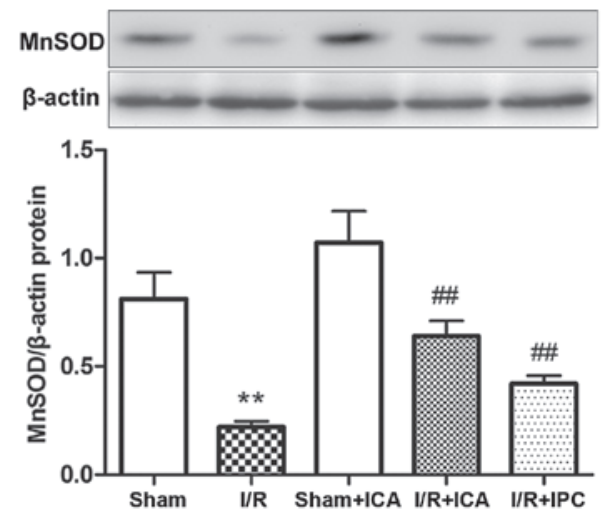

Figure 4. Effects of ICA-mediated SIRT1-FOXO3 pathway activation on the expression of antioxdative factors. (A) Lung MDA activity increased in the I/R group. By contrast, ICA pretreatment or IPC attenuated MDA activity. Values are presented as the mean \pm standard deviation (n=8). (B) Western blot analysis of MnSOD in the lung samples from each group. ICA pretreatment or IPC enhanced MnSOD expression in the lung tissue samples following intestinal I/R. Values are presented as the mean \pm standard deviation $(\mathrm{n}=3)$. ${ }^{* *} \mathrm{P}<0.01$ vs. the control group; ${ }^{\# \#} \mathrm{P}<0.01$ vs. the I/R group. I/R, ischemia/reperfusion; ICA, icariin; IPC, ischemia preconditioning; SIRT1, sirtuin 1; FOXO3, forkhead box protein O3; MDA, malondialdehyde assay; MnSOD, manganese superoxide dismutase.

damage to alveolar cells. As revealed in Fig. 4A, lung MDA activity was increased in the I/R group. By contrast, the results demonstrated that ICA administration and IPC evidently attenuated MDA activity in the I/R+ICA and IPC groups. These results indicated that ICA administration and IPC effectively protected the alveolar cells against oxidative stress in intestinal I/R-induced ALI.

Tofurtherassess the impact of ICA-mediated SIRT1-FOXO3 pathway activation on the expression of antioxidative factors, the role of MnSOD in intestinal I/R-induced ALI was investigated. MnSOD is the primary mitochondrial ROS-scavenging enzyme and is the target protein of the FOXO transcription factors involved in the FOXO3-MnSOD network (21). As revealed in Fig. 4B, MnSOD protein expression in the lung tissue samples was significantly decreased in the intestinal $\mathrm{I} / \mathrm{R}$ group compared with that in the control group, whereas ICA administration or IPC demonstrated an efficient recovery of MnSOD protein levels. These results indicated that ICA administration or IPC upregulated SIRT1 protein expression, which subsequently deacetylated and activated FOXO3, while the latter increased the levels of the antioxidative molecule MnSOD, suggesting that the ICA-mediated activation of the SIRT1-FOXO3 pathway had an antioxdative role in intestinal I/R-induced ALI via MnSOD induction.

\section{Discussion}

Intestinal $\mathrm{I} / \mathrm{R}$ injury is considered a harbinger of systemic inflammation and contributes to distant organ injury and multiple organ dysfunction syndrome (32). The lung is the remote organ most susceptible to injury following intestinal $\mathrm{I} / \mathrm{R}$, which explains why ALI is a major cause of death $(8,9,11)$. Mechanisms underlying ALI are complex, but ultimately induce an antioxidative stress imbalance and the initiation of apoptotic signaling. Previous studies have demonstrated that the antioxidant molecule MnSOD and anti-apoptotic Bcl-2 family of molecules attributed to small intestine I/R-induced ALI (33-35). SIRs were first identified in yeast as silent information regulators and grouped as class III HDACs. SIRs function by removing acetyl groups from lysine residues via NAD consumption. There are seven human SIR homologs (SIRT1-7), each of which exhibits different enzymatic activities and functions, although SIRT1 is the most widely studied $(36,37)$. SIRT1 enhances cellular responses to exogenous stress and survival via regulation of various proteins, including p53, NF- $\mathrm{KB}$ and the FOXO transcription factors $(25,38-40)$. FOXO3 enhances cellular responses to oxidative stress and apoptosis under ischemic or hypoxic conditions (41). In addition, in tissues and organs, IPC has a protective effect via activation of endogenous mechanisms; however, the delayed protection of IPC limits its clinical application. Therefore an ideal method would be to administer drugs, including ICA, which exerted positive effects in the present study, to achieve the effects of IPC.

Following ICA and IPC pretreatment, levels of SIRT1 and acetylated FOXO3 were altered in small intestinal I/R-induced $\mathrm{ALI}$, as well as the regulation of downstream antioxidant factors and apoptosis-associated factors. The present study demonstrated that i) in small intestinal I/R-induced ALI, SIRT1 levels were significantly lower and acetylated FOXO3 levels were significantly higher; ii) following ICA administration or IPC, SIRT1 levels were significantly increased, while acetylated FOXO3 levels were significantly reduced and iii) the protective role of ICA and IPC in this model was associated with the activation of downstream FOXO3-associated molecules, including the antioxidant factor MnSOD, the pro-apoptotic protein Bim and the anti-apoptotic protein Bcl-2. These results suggested that the regulation of the SIRT1/FOXO3 signaling pathway by ICA may be beneficial in intestinal I/R-induced ALI.

Intestinal $\mathrm{I} / \mathrm{R}$ initiates a series of inflammatory and oxidative stress responses (42). In the present study, it was identified that following intestinal $\mathrm{I} / \mathrm{R}$, the intestinal and lung tissues exhibited histological damage and serum TNF- $\alpha$ and IL-6 levels were markedly increased in association with significantly decreased levels of tissue GSH and GSH-PX, and the lung MDA activity was elevated compared with the control group. These changes were parallel to the decrease SIRT1 and MnSOD levels and increased acetylated FOXO3 protein expression. Following ICA pretreatment, SIRT1 mRNA and 
protein levels were elevated, which induced the reduction of acetylated FOXO3 protein levels, which then induced MnSOD production. More importantly, the indicators of injury mentioned above were reversed, which suggested that the SIRT1/FOXO3 signaling pathway had a crucial role in antioxidative stress amelioration following intestinal I/R-induced ALI. Several studies have suggested a functional link between SIRT1 and FOXO3 regulation of the MnSOD-mediated antioxidant pathway regarding ROS production $(41,43)$. In the present study, MnSOD levels were evidently attenuated by intestinal I/R. By contrast, following ICA administration, acetylated FOXO3 expression levels were reduced and MnSOD was upregulated, which was attributed to the increasing levels of deacetylated FOXO3. Therefore, intestinal I/R appeared to activate a cascade resulting in ROS accumulation, SIRT1 reduction, FOXO3 acetylatation and MnSOD downregulation. The regulation of the SIRT1-FOXO3 signaling pathway by ICA upregulated MnSOD expression, thereby strengthening cellular antioxidant capability and survival.

To identify the potential mechanisms of the SIRT1-FOXO3 signaling pathway associated with cell apoptosis in intestinal I/R-induced ALI, the functional link between FOXO3 and apoptosis by evaluating $\mathrm{Bim}$ and $\mathrm{Bcl}-2$ expression was determined using the TUNEL assay to measure the extent of apoptosis. The Bcl-2 family, which has both pro- and anti-apoptotic members, including Bim and Bcl-2, has a critical role in the regulation of cell survival. A study by Dijkers et al (31) suggested that the activation of FOXO3 was sufficient to induce Bim expression. Furthermore, the authors proposed that Bcl-2 suppressed the ability of FOXO3 to induce apoptosis. In addition, a study by Motta et al (24) demonstrated that SIRT1 deacetylated and repressed the activity of FOXO3 to reduce forkhead-dependent apoptosis. The present study demonstrated that Bim expression was increased and Bcl-2 expression was significantly decreased in the intestinal I/R group; however, ICA or IPC pretreatment markedly decreased Bim expression, increased Bcl-2 expression and reduced $\mathrm{FOXO} 3$ acetylation in response to SIRT1 upregulation. Therefore, consistent with the results of previous studies, Bcl-2 overexpression via the SIRT1-FOXO3 signaling pathway protected lung epithelial cells from intestinal I/R-induced apoptosis and subsequent ALI. The TUNEL assay revealed marked alveolar cell apoptosis in the lung tissues of rats in the intestinal I/R group, while alveolar cell apoptosis was markedly attenuated in the ICA and IPC groups.

In conclusion, the present study demonstrated that ICA-mediated SIRT1-FOXO3 signaling regulated oxidative stress injury and cell apoptosis in intestinal I/R-induced ALI. ICA or IPC pretreatment protected the experimental rats from intestinal I/R-induced ALI via activation of the SIRT1-FOXO3 signaling pathway. However, this protective mechanism may be mediated by MnSOD upregulation and enhanced Bcl-2 expression. Manipulation of SIRT1 upregulation may offer a new therapeutic approach to alleviate ALI in intestinal $\mathrm{I} / \mathrm{R}$ patients. It is therefore important to further elucidate the regulation mechanisms of ICA that induced SIRT1 expression and FOXO3 deacetylation. At present, a study is underway in our group to determine the precise mechanisms underlying ICA-mediated regulation in SIRT1 expression.

\section{Acknowledgements}

This study was supported by grants of the Chinese National Natural Science Foundation (no. 81171850) and a Specialized Research Fund by the Doctoral Program of Higher Education of China (no. 20122105110001).

\section{References}

1. Pierro A and Eaton S: Intestinal ischemia reperfusion injury and multisystem organ failure. Semin Pediatr Surg 13: 11-17, 2004.

2. Higuchi S, Wu R, Zhou M, et al: Gut hyperpermeability after ischemia and reperfusion: attenuation with adrenomedullin and its binding protein treatment. Int J Clin Exp Pathol 1: 409-418, 2008.

3. Tendler DA: Acute intestinal ischemia and infarction. Semin Gastrointest Dis 14: 66-76, 2003.

4. Li C and Jackson RM: Reactive species mechanisms of cellular hypoxia-reoxygenation injury. Am J Physiol Cell Physiol 282: C227-C241, 2002.

5. Eltzschig HK and Eckle T: Ischemia and reperfusion - from mechanism to translation. Nat Med 17: 1391-1401, 2011.

6. Cui T, Miksa M, Wu R, et al: Milk fat globule epidermal growth factor 8 attenuates acute lung injury in mice after intestinal ischemia and reperfusion. Am J Respir Crit Care Med 181: 238-246, 2010.

7. Wu R, Dong W, Ji Y, et al: Orexigenic hormone ghrelin attenuates local and remote organ injury after intestinal ischemia-reperfusion. PLOS One 3: e2026, 2008.

8. Sorkine P, Szold O, Halpern P, et al: Gut decontamination reduces bowel ischemia-induced lung injury in rats. Chest 112: 491-495, 1997.

9. Mura M, Andrade CF, Han B, et al: Intestinal ischemia-reperfusion induced acute lung injury and oncotic cell death in multiple organs. Shock 28: 227-238, 2007.

10. Fu TL, Zhang WT, Zhang L, et al: L-arginine administration ameliorates serum and pulmonary cytokine response after gut ischemia-reperfusion in immature rats. World J Gastroenterol 11: 1070-1072, 2005.

11. Moraes LB, Murakami AH, Fontes B, et al: Gut ischemia/reperfusion induced acute lung injury is an alveolar macrophage dependent event. J Trauma 64: 1196-1200, 2008.

12. Ben DF, Yu XY, Ji GY, et al: TLR4 mediates lung injury and inflammation in intestinal ischemia-reperfusion. J Surg Res 174: 326-333, 2012.

13. Biswas SK and Rahman I: Environmental toxicity, redox signaling and lung inflammation: the role of glutathione. Mol Aspects Med 30: 60-76, 2009.

14. Marfe G, Tafani M, Pucci B, et al: The effect of marathon on mRNA expression of anti-apoptotic and pro-apoptotic proteins and sirtuins family in male recreational long-distance runners. BMC Physiol 10: 7, 2010.

15. Lai L, Yan L, Gao S, et al: Type 5 adenylyl cyclase increases oxidative stress by transcriptional regulation of MnSOD via the sirt1/foxo3a pathway. Circulation 127: 1692-1701, 2013.

16. Alcendor RR, Kirshenbaum LA, Imai S, et al: Silent information regulator 2alpha, a longevity factor and class III histone deacetylase, is an essential endogenous apoptosis inhibitor in cardiac myocytes. Circ Res 95: 971-980, 2004.

17. Kelly G: A review of the sirtuin system, its clinical implications, and the potential role of dietary activators like resveratrol: part 1. Altern Med Rev 15: 245-263, 2010.

18. Sauve AA, Wolberger C, Schramm VL and Boeke JD: The biochemistry of sirtuins. Ann Rev Biochem 75: 435-465, 2006.

19. Haiqis MC and Guarente LP: Mammalian sirtuins - emerging roles in physiology, aging, and calorie restriction. Gen Dev 20: 2913-2921, 2006.

20. Hsu CP, Zhai P, Yamamoto T, et al: Silent information regulator 1 protects the heart from ischemia/reperfusion. Circulation 122: 2170-2182, 2010

21. Brunet A, Sweeney LB, Sturgill JF, et al: Stress-dependent regulation of FOXO transcription factors by the SIRT1 deacetylase. Science 303: 2011-2015, 2004.

22. Langley E, Pearson M,Faretta M, et al: Human SIR2 deacetylates p53 and antagonizes PML/p53-induced cellular senescence. EMBO J 21: 2383-2396, 2002. 
23. Yeung F, Hoberg JE, Ramsey CS, et al: Modulation of NF-kappaB-dependent transcription and cell survival by the SIRT1 deacetylase. EMBO J 23: 2369-2380, 2004.

24. Motta MC, Divecha N, Lemieux M, et al: Mammalian SIRT1 represses forkhead transcription factors. Cell 116: 551-563, 2004

25. Picone P, Giacomazza D, Vetri V, et al: Insulin-activated Akt rescues $A \beta$ oxidative stress-induced cell death by orchestrating molecular trafficking. Aging Cell 10: 832-843, 2011.

26. Wang L, Zhang L, Chen ZB, et al: Icariin enhances neuronal survival after oxygen and glucose deprivation by increasing SIRT1. Eur J Pharmacol 609: 40-44, 2009.

27. Zhang L, Huang S, Chen Y, et al: Icariin inhibits hydrogen peroxide-mediated cytotoxicity by up-regulating sirtuin type 1-dependent catalase and peroxiredoxin. Basic Clin Pharmacol Toxicol 107: 899-905, 2010.

28. Zhu HR, Wang ZY, Zhu XL, et al: Icariin protects against brain injury by enhancing SIRT1-dependent PGC-1alpha expression in experimental stroke. Neuropharmacology 59: 70-76, 2010.

29. Wang GZ, Yao JH, Jing HR, et al: Suppression of the p66shc adapter protein by protocatechuic acid prevents the development of lung injury induced by intestinal ischemia reperfusion in mice. J Trauma Acute Care Surg 73: 1130-1137, 2012.

30. Mikawa K, Nishina K, Takao Y and Obara H: ONO-1714, a nitric oxide synthase inhibitor, attenuates endotoxin-induced acute lung injury in rabbits. Anesth Analg 97: 1751-1755, 2003.

31. Dijkers PF, Medema RH, Lammers JW, et al: Expression of the pro-apoptotic Bcl-2 family member Bim is regulated by the forkhead transcription factor FKHR-L1. Current Biology 10 : 1201-1204, 2000.

32. Hassoun HT, Kone BC, Mercer DW, et al: Post-injury multiple organ failure: the role of the gut. Shock 15: 1-10,2001.

33. Kostopanagiotou G, Avgerinos E, Costopanaqiotou C, et al: Acute lung injury in a rat model of intestinal ischemia-reperfusion: the potential time depended role of phospholipases A (2). J Surg Res 147: 108-116, 2008
34. Yeh KY, Yeh M, Glass J, et al: Rapid activation of NF-kappaB and AP-1 and target gene expression in postischemic rat intestine. Gastroenterology 118: 525-534, 2000.

35. Koo HC, Davis JM, Li Y, et al: Effects of transgene expression of superoxide dismutase and glutathione peroxidase on pulmonary epithelial cell growth in hyperoxia. Am J Physiol Lung Cell Mol Physiol 288: L718-L726, 2005.

36. Blander G and Guarente L: The sir2 family of protein deacetylases. Annu Rev Biochem 73: 417-435, 2004.

37. North BJ and Verdin E: Sirtuins: Sir2-related NAD-dependent protein deacetylases. Genome Biol 5: 224, 2004.

38. Okawa H, Motohashi H, Kobayashi A, et al: Hepatocyte-specific deletion of the keapl gene activates Nrf2 and confers potent resistance against acute drug toxicity. Biochem Biophys Res Commun 339: 79-88, 2006.

39. Reisman SA, Csanaky IL, Aleksunes LM and Klaassen CD: Altered disposition of acetaminophen in Nrf2-null and Keapl-knockdown mice. Toxicol Sci 109: 31-40, 2009.

40. Zhai X, Lin M,Zhang F, et al: Dietary flavonoid genistein induces Nrf2 and phase II detoxification gene expression via ERKs and PKC pathways and protects against oxidative stress in Caco-2 cells. Mol Nutr Food Res 57: 249-259, 2013.

41. Calvert JW, Jha S, Gundewar S, et al: Hydrogen sulfide mediates cardioprotection through Nrf2 signaling. Circ Res 105: 365-374, 2009.

42. Li Y, Yao JH, Hu XW, et al: Inhibition of Rho kinase by fasudil hydrochloride attenuates lung injury induced by intestinal ischemia and reperfusion. Life Sci 88: 104-109, 2011.

43. Tanaka J, Qiang L, Banks AS, et al: Foxo1 links hyperglycemia to LDL oxidation and endothelial nitric oxide synthase dysfunction in vascular endothelial cells. Diabetes 58: 2344-2354, 2009. 